\title{
UNIFORM ESTIMATES FOR THE SOLUTIONS OF THE SCHRÖDINGER EQUATION ON THE TORUS AND REGULARITY OF SEMICLASSICAL MEASURES
}

\author{
Tayeb Aïssiou, Dmitry Jakobson, And Fabricio Macià
}

\begin{abstract}
We establish uniform bounds for the solutions $\mathrm{e}^{i t \Delta} u$ of the Schrödinger equation on arithmetic flat tori, generalizing earlier results by J. Bourgain. We also study the regularity properties of weak-* limits of sequences of densities of the form $\left|\mathrm{e}^{i t \Delta} u_{n}\right|^{2}$ corresponding to highly oscillating sequences of initial data $\left(u_{n}\right)$. We obtain improved regularity properties of those limits using previous results by N. Anantharaman and F. Macià on the structure of semiclassical measures for solutions to the Schrödinger equation on the torus.
\end{abstract}

\section{Introduction}

Consider the Schrödinger flow $\mathrm{e}^{i t \Delta}$ on the arithmetic flat $d$-dimensional torus $\mathbb{T}^{d}:=$ $\mathbb{R}^{d} / 2 \pi \mathbb{Z}^{d}$. That is, given any $u \in L^{2}\left(\mathbb{T}^{d}\right)$, the function $\psi(x, t):=\mathrm{e}^{i t \Delta} u(x)$ is the solution to:

$$
i \partial_{t} \psi(x, t)+\Delta \psi(x, t)=0, \quad \psi(\cdot, 0)=u .
$$

In this paper, we are be interested in those regularity properties of $\psi$ that can be expressed through the quantity:

$$
|\psi(x, t)|^{2}
$$

which a priori, is a continuous function of $t \in \mathbb{T}$ (as the Schrödinger flow is periodic) taking values in the set of positive elements in $L^{1}\left(\mathbb{T}^{d}\right)$. By conservation of energy, the total mass of $|\psi(\cdot, t)|^{2}$ equals $\|u\|_{L^{2}\left(\mathbb{T}^{d}\right)}^{2}$ for every $t \in \mathbb{T}$.

Given $u \in L^{2}\left(\mathbb{T}^{d}\right)$, denote by $b_{u}(l, s)$, with $(l, s) \in \mathbb{Z}^{d} \times \mathbb{Z}$, the Fourier coefficients of $|\psi|^{2}$ :

$$
\left|e^{i t \Delta} u(x)\right|^{2}=\sum_{(l, s) \in \mathbb{Z}^{d} \times \mathbb{Z}} b_{u}(l, s) \mathrm{e}^{i(l, s) \cdot(x, t)} ;
$$

note that $b_{u}$ is a quadratric quantity in $u$. We shall prove the following.

Theorem 1.1. There exists a constant $C_{d}>0$ such that

$$
\left\|b_{u}\right\|_{l^{d+1}\left(\mathbb{Z}^{d+1}\right)} \leq C_{d}\|u\|_{L^{2}\left(\mathbb{T}^{d}\right)}^{2},
$$

for every $u \in L^{2}\left(\mathbb{T}^{d}\right)$.

Received by the editors April 11, 2012.

1991 Mathematics Subject Classification. 58G25, 81Q50, 35Q41, 35B45, 42B05.

Key words and phrases. semiclassical limits, linear Schrödinger equation on a torus, quantum limits, semiclassical measures, dispersive estimates. 
When $d=1$, Theorem 1.1 has been proved by Bourgain [3], using the methods developed by Cooke [6] and Zygmund [12]. Note that in that case, estimate (1.2) is equivalent to:

$$
\left\|\mathrm{e}^{i t \partial_{x}^{2}} u\right\|_{L^{4}\left(\mathbb{T}_{x} \times \mathbb{T}_{t}\right)} \leq\left(C_{1}\right)^{1 / 2}\|u\|_{L^{2}(\mathbb{T})} .
$$

When $d \geq 2$, Bourgain has proved in $[3,5]$ frequency-dependent generalizations of estimate (1.3) that are optimal in many cases. When the initial datum $u(x)$ is an eigenfunction of $\Delta$ :

$$
\Delta u+\lambda u=0, \quad\|u\|_{L^{2}\left(\mathbb{T}^{d}\right)}=1
$$

then $\mathrm{e}^{i t \Delta} u=\mathrm{e}^{-i t \lambda} u$, which implies $\left|\mathrm{e}^{i t \Delta} u\right|^{2}=|u|^{2}$. For that class of initial data, the Fourier coefficients $b_{u}(l)$ only depend on $l \in \mathbb{Z}^{d}$ and the exponent in estimate (1.2) can be improved to:

$$
\left\|b_{u}\right\|_{l^{d}\left(\mathbb{Z}^{d}\right)} \leq K_{d}
$$

where the constant $K_{d}$ is independent of the eigenvalue $\lambda$. This result was established in $[6,12]$ for $d=2$ and in $[8,11,1]$ for $d \geq 3$.

Theorem 1.1 shows that as a function of space and time, $\mathrm{e}^{i t \Delta} u$ is more regular than being merely a function in $C\left(\mathbb{T} ; L^{2}\left(\mathbb{T}^{d}\right)\right.$ ), but this regularity is only expressed in terms of the summability properties of the Fourier coefficients of its modulus square. The proof of Theorem 1.1 relies on ideas in [8]; it is presented in Section 2, along with a straightforward generalization to the density-matrix Schrödinger equation (Theorem 2.2).

Next, we state our second result. Suppose $\left(u_{n}\right)$ is a bounded sequence in $L^{2}\left(\mathbb{T}^{d}\right)$. Then the sequence

$$
\left(\left|\mathrm{e}^{i t \Delta} u_{n}\right|^{2}\right) \subset C\left(\mathbb{T} ; L^{1}\left(\mathbb{T}^{d}\right)\right),
$$

is uniformly bounded. It is always possible to extract a subsequence $\left(u_{n^{\prime}}\right)$ such that that the $\left|\mathrm{e}^{i t \Delta} u_{n^{\prime}}\right|^{2}$ converge to some positive measure $\nu \in L^{\infty}\left(\mathbb{T} ; \mathcal{M}_{+}\left(\mathbb{T}^{d}\right)\right)$ in the weak-* topology, ${ }^{1}$ i.e.,

$$
\lim _{n^{\prime} \rightarrow \infty} \int_{\mathbb{T} \times \mathbb{T}^{d}} \phi(t) a(x)\left|\mathrm{e}^{i t \Delta} u_{n^{\prime}}(x)\right|^{2} d t d x=\int_{\mathbb{T}} \int_{\mathbb{T}^{d}} \phi(t) a(x) \nu(d x, t) d t,
$$

for every $\phi \in L^{1}(\mathbb{T})$ and every $a \in C\left(\mathbb{T}^{d}\right)$. In what follows, we shall say that a measure $\nu$ is a weak-* accumulation point of the sequence of densities $\left(\left|\mathrm{e}^{i t \Delta} u_{n}\right|^{2}\right)$ if (1.6) holds for some subsequence.

Our next result deals with the regularity properties of those measures $\nu$ that arise as weak-* accumulation points of sequences of densities of orbits of the Schrödinger flow. A result by Bourgain [4] asserts that in fact all weak-* accumulation points $\nu$ are absolutely continuous with respect to the Lebesgue measure, i.e., one always has $\nu \in L^{\infty}\left(\mathbb{T} ; L^{1}\left(\mathbb{T}^{d}\right)\right)$. This result was further generalized by Anantharaman and Macià [2] to the case of the Laplacian plus a potential; moreover, these authors deduced a propagation law for $\nu(\cdot, t)$ and clarified the dependence of $\nu$ on the sequence of initial data $\left(u_{n}\right)$ (these results generalize those in [10]).

In addition, because of estimate (1.2) one easily sees that such a $\nu$ also satisfies $\|\widehat{\nu}\|_{l^{d+1}\left(\mathbb{Z}^{d+1}\right)}<\infty$, where $\widehat{\nu}(l, s),(l, s) \in \mathbb{Z}^{d} \times \mathbb{Z}$, stand for the Fourier coefficients of $\nu$. These two facts express that the regularity properties of $\nu$ are as good as those of the

\footnotetext{
${ }^{1}$ We have denoted by $\mathcal{M}_{+}\left(\mathbb{T}^{d}\right)$ the set of positive Radon measures on $\mathbb{T}^{d}$.
} 
densities $\left|\mathrm{e}^{i t \Delta} u_{n}\right|^{2}$. And this cannot be improved at this level of generality, since if a sequence $\left(u_{n}\right)$ converges strongly to some $u$ in $L^{2}\left(\mathbb{T}^{d}\right)$ then $\nu(\cdot, t)=\left|\mathrm{e}^{i t \Delta} u\right|^{2}$.

However, the regularity of the weak-* accumulation points can be improved if we restrict ourselves to sequences $\left(u_{n}\right)$ that are highly oscillating. This is for instance the case when $\left(u_{n}\right)$ consists of eigenfunctions of the Laplacian (1.4) corresponding to eigenvalues $\lambda=\lambda_{n} \rightarrow \infty$ as $n \rightarrow \infty$. If

$$
\nu(x)=\sum_{l \in \mathbb{Z}^{d}} \widehat{\nu}(l) \mathrm{e}^{i l \cdot x}
$$

is an accumulation point of $\left(\left|\mathrm{e}^{i t \Delta} u_{n}\right|^{2}\right)=\left(\left|u_{n}\right|^{2}\right)$ then $\nu$ is more regular than a priori expected. It is constant if $d=1$; it is a trigonometric polynomial if $d=2$, as shown by Jakobson [8]; and for $d \geq 3$ it satisfies (see again [8]):

$$
\|\widehat{\nu}\|_{l^{d-2}\left(\mathbb{Z}^{d}\right)}<\infty
$$

which is an improvement by two in the summability exponent with respect to the corresponding estimate (1.5) for eigenfunctions.

Here we shall deal with sequences of initial data that are more general than eigenfunctions but still exhibit oscillating behavior around some characteristic frequencies. More precisely, we shall assume that the sequence of initial data $\left(u_{n}\right)$ satisfies the following condition:

(S) There exists a sequence of positive reals $\left(h_{n}\right)$ that tends to zero such that:

and

$$
\limsup _{n \rightarrow \infty} \sum_{|l|<\delta / h_{n}}\left|\widehat{u_{n}}(l)\right|^{2} \rightarrow 0, \quad \text { as } \delta \rightarrow 0^{+},
$$

$$
\limsup _{n \rightarrow \infty} \sum_{|l|>R / h_{n}}\left|\widehat{u_{n}}(l)\right|^{2} \rightarrow 0, \quad \text { as } R \rightarrow \infty .
$$

Above, $\widehat{u_{n}}(l), l \in \mathbb{Z}^{d}$, are the Fourier coefficients of $u_{n}$. This condition ensures that the $L^{2}$-norm of $u_{n}$ asymptotically localized on frequencies of the order of $1 / h_{n}$. Condition (S) has been introduced in [7] (in the context of $\mathbb{R}^{d}$ ) under the name of $\left(h_{n}\right)$-oscillation. We refer the reader to example 2.3 of [7] for the construction of a sequence weakly converging to zero in $L^{2}\left(\mathbb{R}^{d}\right)$ such that the Euclidean version of property $(\mathbf{S})$ fails.

Theorem 1.2. Suppose that $\left(u_{n}\right)$ is a bounded sequence in $L^{2}\left(\mathbb{T}^{d}\right)$ that satisfies condition $(\boldsymbol{S})$ above. Let $\nu \in L^{\infty}\left(\mathbb{T} ; L^{1}\left(\mathbb{T}^{d}\right)\right)$ be a weak-* accumulation point of $\left(\left|\mathrm{e}^{i t \Delta} u_{n}\right|^{2}\right)$. If $\widehat{\nu}(l, s),(l, s) \in \mathbb{Z}^{d} \times \mathbb{Z}$ are the Fourier coefficients of $\nu(t, x)$ then:

$$
\|\widehat{\nu}\|_{l^{d}\left(\mathbb{Z}^{d+1}\right)}<\infty .
$$

Therefore, if one imposes condition $(\mathbf{S})$ on the sequence of initial data, the accumulation points $\nu$ enjoy more regularity than that a priori expressed by Theorem 1.1. The proof of this result is presented in Section 3; it is based on two ingredients: estimate (1.2) in its matrix-density version; second, the results on the structure of semiclassical measures for the Schrödinger flow in $[10,2]$ that allow us to lower the dimension by one.

If one consider sequences of initial data that satisfy conditions that are more restrictive than $(\mathbf{S})$ it is possible to show additional regularity properties on the 
corresponding weak-* accumulation points. This is the scope of Theorem 3.1, which is stated and proved in the second part of Section 3.

\section{Uniform estimates for the Schrödinger flow}

We first prove Theorem 1.1; then we state a straightforward generalization of this result to solutions to the density-matrix Schrödinger equation that will be used in the proof of Theorem 1.2.

2.1. Proof of Theorem 1.1. Let $u \in L^{2}\left(\mathbb{T}^{d}\right)$ given by

$$
u(x)=\sum_{k \in \mathbb{Z}^{d}} a_{k} \mathrm{e}^{i k \cdot x}
$$

to lighten our writing, we shall use the following normalization of the $L^{2}$-norm:

$$
\|u\|_{L^{2}\left(\mathbb{T}^{d}\right)}^{2}:=\sum_{k \in \mathbb{Z}^{d}}\left|a_{k}\right|^{2}=\int_{\mathbb{T}^{d}}|u(x)|^{2} \frac{d x}{(2 \pi)^{d}} .
$$

Consider the following set of lattice points:

$$
\mathcal{P}:=\left\{\left(k,-|k|^{2}\right): k \in \mathbb{Z}^{d}\right\} \subset \mathbb{Z}^{d+1} ;
$$

this is precisely the set of lattice points contained in the paraboloid of $\mathbb{R}^{d+1}$ obtained as the graph of $-|x|^{2}$ for $x \in \mathbb{R}^{d}$. We shall denote a generic point in $\mathbb{Z}^{d+1}$ as $(k, n)$ with $k \in \mathbb{Z}^{d}$ and $n \in \mathbb{Z}$.

The orbit of the Schrödinger flow corresponding to $u$ is:

$$
\mathrm{e}^{i t \Delta} u(x)=\sum_{k \in \mathbb{Z}^{d}} a_{k} \mathrm{e}^{i\left(k \cdot x-|k|^{2} t\right)}=\sum_{(k, n) \in \mathcal{P}} a_{k} \mathrm{e}^{i(k, n) \cdot(x, t)},
$$

and,

$$
\left|\mathrm{e}^{i t \Delta} u(x)\right|^{2}=\sum_{(l, s) \in \mathbb{Z}^{d+1}} b_{u}(l, s) \mathrm{e}^{i(l, s) \cdot(x, t)},
$$

where, for $(l, s) \in \mathbb{Z}^{d+1}$ we have set:

$$
b_{u}(l, s):=\sum_{\begin{array}{c}
k-j=l, \\
|j|^{2}-|k|^{2}=s
\end{array}} a_{k} \overline{a_{j}}=\sum_{\begin{array}{c}
(k, n)-(j, m)=(l, s), \\
(k, n),(j, m) \in \mathcal{P}
\end{array}} a_{k} \overline{a_{j}} ;
$$

note that the range of the sum above might be empty, in which case we set $b_{u}(l, s):=0$. Clearly

$$
b_{u}(0,0)=\|u\|_{L^{2}\left(\mathbb{T}^{d}\right)}^{2}, \quad b_{u}(0, s)=0 \text { for } s \in \mathbb{Z} \backslash\{0\},
$$

We start by making some elementary geometric remarks. Let $j \in \mathbb{Z}^{d}$ and suppose that there exist $(l, s) \in \mathbb{Z}^{d+1} \backslash\{(0,0)\}$ and $k \in \mathbb{Z}^{d}$ such that $k-j=l$ and $|j|^{2}-|k|^{2}=s$. This is the same as saying that $(l, s)$ is a chord of the discrete paraboloid $\mathcal{P}$ with origin at $\left(j,-|j|^{2}\right)$. We can rewrite the condition on the squares of the lengths of $k$ and $j$ as:

$$
s=-l \cdot(k+j)=-l \cdot(l+2 j)=-|l|^{2}-2 l \cdot j .
$$

Therefore, the set of all $j \in \mathbb{Z}^{d}$ such that $\left(j,-|j|^{2}\right)$ is the origin of a chord $(l, s)$ on the paraboloid $\mathcal{P}$ is equal to

$$
H_{(l, s)}:=\left\{j \in \mathbb{Z}^{d}: j \cdot \frac{l}{|l|}=-\frac{1}{2}\left(\frac{s}{|l|}+|l|\right)\right\},
$$


which is the set of lattice points that lie on a certain hyperplane of $\mathbb{R}^{d}$ which is orthogonal to $l$ and at whose distance from the origin is determined by $|l|$ and $s$. Note in particular that

$$
H_{(l, s)} \cap H_{\left(l, s^{\prime}\right)} \neq \emptyset \Leftrightarrow s=s^{\prime} .
$$

We summarize the preceding geometric discussion as:

Let $(k, n),(j, m) \in \mathcal{P}$ and $(l, s) \in \mathbb{Z}^{d+1}$ with $l \neq 0$. That $(k, n)-(j, m)=(l, s)$ is equivalent to $j \in H_{(l, s)}$ where $s$ is given by (2.4) and $k=j+l$.

Now, by (2.3),

$$
\sum_{(l, s) \in \mathbb{Z}^{d+1}}\left|b_{u}(l, s)\right|^{d+1}=\|u\|_{L^{2}\left(\mathbb{T}^{d}\right)}^{2(d+1)}+\sum_{(l, s) \in \mathbb{Z}^{d+1}, l \neq 0}\left|b_{u}(l, s)\right|^{d+1} .
$$

Our goal will be to estimate the second term in the above sum by $\|u\|_{L^{2}\left(\mathbb{T}^{d}\right)}^{2(d+1)}$. We have proved above that, for $l \neq 0$, we can rewrite $(2.2)$ as:

$$
b_{u}(l, s)=\sum_{j \in H_{(l, s)}} a_{j+l} \overline{a_{j}},
$$

and therefore,

$$
\left|b_{u}(l, s)\right|^{d+1} \leq \sum_{j_{1}, \ldots, j_{d+1} \in H_{(l, s)}} \prod_{\sigma=1}^{d+1}\left|a_{j_{\sigma}+l} a_{j_{\sigma}}\right| .
$$

Let $\delta \geq 0$ be an integer. For any $r \in\{0,1, \ldots, \delta-1\}$ denote by $\mathcal{V}_{r}^{\delta}$ the set of the $\left(j_{1}, \ldots, j_{\delta+1}\right) \in \mathbb{Z}^{d(\delta+1)}$ such that $j_{1}, \ldots, j_{\delta+1}$ span an affine variety in $\mathbb{R}^{d}$ of dimension $r$. We can rewrite (2.6) as:

$$
\left|b_{u}(l, s)\right|^{d+1} \leq \sum_{r=0}^{d-1} \sum_{\left(j_{1}, \ldots, j_{d+1}\right) \in\left(H_{(l, s)}\right)^{d+1} \cap \mathcal{V}_{r}^{d}} \prod_{\sigma=1}^{d+1}\left|a_{j_{\sigma}+l} a_{j_{\sigma}}\right| .
$$

Therefore, in order to have a bound for $\left\|b_{u}\right\|_{l^{d+1}}^{d+1}$ it suffices to estimate, for each $r=0,1, \ldots, d-1$, the term:

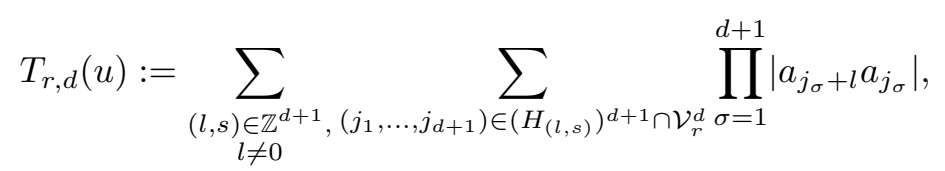

which involves only indices $j_{1}, \ldots, j_{d+1}$ that span an affine variety of dimension $r$.

This is a consequence of the next lemma, which is slightly more general than what is needed at this point, but that will also be needed in the proof of Theorem 2.2 in the next paragraph.

Lemma 2.1. Let $\delta$ and $r$ be integers with $0 \leq \delta \leq d$ and $0 \leq r \leq \delta-1$. For every $u \in L^{2}\left(\mathbb{T}^{d}\right)$ given by $u(x)=\sum_{k \in \mathbb{Z}^{d}} a_{k} \mathrm{e}^{i k \cdot x}$ define:

$$
T_{r, \delta}(u):=\sum_{\substack{(l, s) \in \mathbb{Z}^{d+1} \\ l \neq 0}} \sum_{\left(j_{1}, \ldots, j_{\delta+1}\right) \in\left(H_{(l, s)}\right)^{\delta+1} \cap \mathcal{V}_{r}^{\delta}} \prod_{\sigma=1}^{\delta+1}\left|a_{j_{\sigma}+l} a_{j_{\sigma}}\right| .
$$


Then

$$
T_{r, \delta}(u) \leq\left(\begin{array}{c}
\delta \\
r
\end{array}\right)\|u\|_{L^{2}\left(\mathbb{T}^{d}\right)}^{2(\delta+1)} .
$$

Proof. The case $r=0$ is simple to estimate. Note that given $(k, j) \in \mathbb{Z}^{d} \times \mathbb{Z}^{d}$ with $k \neq j$ there exist a unique $(l, s) \in \mathbb{Z}^{d+1}$ with $l \neq 0$ such that $j \in H_{(l, s)}$ and $k=j+l$. Therefore:

$$
\begin{aligned}
T_{0, \delta}(u) & =\sum_{\substack{(l, s) \in \mathbb{Z}^{d+1} \\
l \neq 0}} \sum_{j \in H_{(l, s)}}\left|a_{j+l} a_{j}\right|^{\delta+1} \\
& =\sum_{\substack{(k, j) \in \mathbb{Z}^{d} \times \mathbb{Z}^{d} \\
k \neq j}}\left|a_{k} a_{j}\right|^{\delta+1} \leq\left(\sum_{j \in \mathbb{Z}^{d}}\left|a_{j}\right|^{\delta+1}\right)^{2} \leq\|u\|_{L^{2}\left(\mathbb{T}^{d}\right)}^{2(\delta+1)} .
\end{aligned}
$$

Now suppose $1 \leq r \leq \delta-1$ and consider a fixed summand in (2.8):

$$
\prod_{\sigma=1}^{\delta+1}\left|a_{j_{\sigma}+l} a_{j_{\sigma}}\right|
$$

corresponding to some $\left(j_{1}, \ldots, j_{\delta+1}\right) \in \mathcal{V}_{r}^{\delta}$; denote by $\mathcal{L}$ the $r$-dimensional affine variety spanned by the $j_{\sigma}$ 's. Choose the least indices $1=\alpha_{1}<\cdots<\alpha_{r+1} \leq \delta+1$ such that $j_{\alpha_{1}}, \ldots, j_{\alpha_{r+1}}$ span $\mathcal{L}$, and denote by $\beta_{1}<\cdots<\beta_{\delta-r}$ the remaining indices. We now estimate (2.9) by:

$$
\frac{1}{2}\left(\prod_{i=1}^{r+1}\left|a_{j_{\alpha_{i}}}\right|^{2} \prod_{i=1}^{\delta-r}\left|a_{j_{\beta_{i}}+l}\right|^{2}+\prod_{i=1}^{r+1}\left|a_{j_{\alpha_{i}}+l}\right|^{2} \prod_{i=1}^{\delta-r}\left|a_{j_{\beta_{i}}}\right|^{2}\right) .
$$

We shall perform our analysis focusing on the first summand. It will be clear that the second summand can be dealt with in a completely analogous manner.

Given $\left(j_{1}, \ldots, j_{\delta+1}\right) \in \mathcal{V}_{r}^{\delta}$ we define $\left(\tilde{j}_{1}, \ldots, \tilde{j}_{\delta+1}\right) \in \mathcal{V}_{r+1}^{\delta}$ as follows: set $\tilde{j}_{i}=j_{\alpha_{i}}$ for $i=1, \ldots, r+1$ and $\tilde{j}_{i}=j_{\beta_{i}}+l$ for $i=r+2, \ldots, \delta+1$. Thus, we can put the first summand in (2.10) as:

$$
\prod_{i=1}^{\delta+1}\left|a_{\tilde{j}_{i}}\right|^{2}, \quad \text { for some }\left(\tilde{j}_{1}, \ldots, \tilde{j}_{\delta+1}\right) \in \mathcal{V}_{r+1}^{\delta} .
$$

In order to estimate $T_{r, \delta}(u)$ by a sum of terms of this form over indices in $\mathcal{V}_{r+1}^{\delta}$ we should take into account the following:

1. A $(\delta+1)$-tuple $\left(\tilde{j}_{1}, \ldots, \tilde{j}_{\delta+1}\right) \in \mathcal{V}_{r+1}^{\delta}$ is obtained from $\left(j_{1}, \ldots, j_{\delta+1}\right) \in \mathcal{V}_{r}^{\delta}$ by applying a permutation that maps monotonically the indices $1=\alpha_{1}<\cdots<\alpha_{r+1}$ into $1, \ldots, r+1$ and the indices $\beta_{1}, \ldots, \beta_{\delta-r}$ into $r+2, \ldots, \delta+1$. Note that there are $\left(\begin{array}{l}\delta \\ r\end{array}\right)$ such permutations.

2. Suppose $\left(\tilde{j}_{1}, \ldots, \tilde{j}_{\delta+1}\right)$ is obtained from $\left(j_{1}, \ldots, j_{\delta+1}\right) \in \mathcal{V}_{r}^{\delta}$. Then there exists a unique $(l, s) \in \mathbb{Z}^{d+1}, l \neq 0$, such that $\left(j_{1}, \ldots, j_{\delta+1}\right) \in\left(H_{(l, s)}\right)^{\delta+1}$. This is due to the fact that that $l$ is a direction contained in the $(r+1)$-dimensional affine variety spanned by $\tilde{j}_{1}, \ldots, \tilde{j}_{r+2}$ and that $l$ must be orthogonal to

$$
\mathcal{L}:=\operatorname{span}\left\{j_{1}, \ldots, j_{\delta+1}\right\}=\operatorname{span}\left\{\tilde{j}_{1}, \ldots, \tilde{j}_{r+1}\right\} .
$$


Therefore, it must coincide with the orthogonal projection of, say, $\tilde{j}_{r+2}-\tilde{j}_{1}$ onto the variety that is orthogonal to $\mathcal{L}$ and contains $j_{1}=\tilde{j}_{1}$. Once $l$ is determined uniquely so is $s$, by (2.4).

As a consequence of this, we deduce the bound:

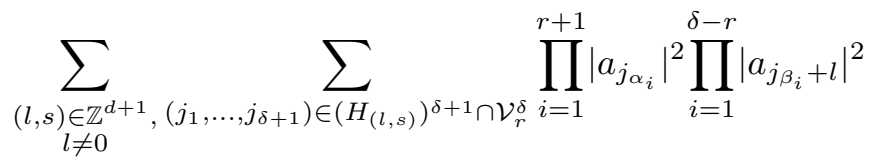

$$
\begin{aligned}
& \leq\left(\begin{array}{l}
\delta \\
r
\end{array}\right) \sum_{\left(\tilde{j}_{1}, \ldots, \tilde{j}_{\delta+1}\right) \in \mathcal{V}_{r+1}^{\delta}} \prod_{\sigma=1}^{\delta+1}\left|a_{\tilde{j}_{\sigma}}\right|^{2} \leq\left(\begin{array}{l}
\delta \\
r
\end{array}\right)\|u\|_{L^{2}\left(\mathbb{T}^{d}\right)}^{2(\delta+1)} .
\end{aligned}
$$

The same estimate can be established for the sums corresponding to the second summands in (2.10); therefore:

$$
T_{r, \delta}(u) \leq\left(\begin{array}{l}
\delta \\
r
\end{array}\right)\|u\|_{L^{2}\left(\mathbb{T}^{d}\right)}^{2(\delta+1)}
$$

Now, applying Lemma 2.1 with $\delta=d$ we obtain:

$$
\sum_{(l, s) \in \mathbb{Z}^{d+1}}\left|b_{u}(l, s)\right|^{d+1}=\|u\|_{L^{2}\left(\mathbb{T}^{d}\right)}^{2(d+1)}+\sum_{r=0}^{d-1} T_{r, d}(u) \leq 2^{d}\|u\|_{L^{2}\left(\mathbb{T}^{d}\right)}^{2(d+1)},
$$

which concludes the proof of Theorem 1.1.

2.2. Estimates for the density-matrix Schrödinger equation. Denote by $\mathcal{L}_{+}^{1}$ the set of symmetric, non-negative, trace-class operators on $L^{2}\left(\mathbb{T}^{d}\right)$. Every operator $A \in \mathcal{L}_{+}^{1}$ is defined by an integral kernel $\rho \in L^{2}\left(\mathbb{T}^{d} \times \mathbb{T}^{d}\right)$,

$$
\rho(x, y)=\sum_{k, j} \widehat{\rho}_{k, j} \mathrm{e}^{i k \cdot x} \mathrm{e}^{-i j \cdot y} .
$$

The self-adjointness of $A$ implies that $\rho$ is symmetric:

$$
\rho(x, y)=\overline{\rho(y, x)},
$$

and from the fact that $A$ is non-negative and trace-class we deduce that

$$
t_{\rho}(x):=\sum_{k, j} \widehat{\rho}_{k, j} \mathrm{e}^{i(k-j) \cdot x}
$$

is a non-negative function in $L^{1}\left(\mathbb{T}^{d}\right)$.

When no confusion arises, we shall use $\rho$ to refer both to a generic operator in $\mathcal{L}_{+}^{1}$ and to its integral kernel. Note that, since operators $\rho \in \mathcal{L}_{+}^{1}$ are non-negative and symmetric, their trace norm is given by:

$$
\|\rho\|_{\mathcal{L}^{1}}:=\operatorname{Tr}(\rho)=\left\|t_{\rho}\right\|_{L^{1}\left(\mathbb{T}^{d}\right)} .
$$

Moreover, if $\rho(x, y)=u(x) \overline{u(y)}$ is the orthogonal projection on a function $u \in L^{2}\left(\mathbb{T}^{d}\right)$ then $\rho \in \mathcal{L}_{+}^{1}$ and $t_{\rho}=|u|^{2}$. Usually, one refers to the elements of $\mathcal{L}_{+}^{1}$ as density matrices. 
The Schrödinger equation for density matrices (also known as the Heisenberg-Von Neumann equation) is:

$$
i \partial_{t} A(t)+[\Delta, A(t)]=0, \quad A(0)=\rho \in \mathcal{L}_{+}^{1},
$$

where $[\cdot, \cdot]$ denotes the commutator bracket. Clearly,

$$
A(t)=\mathrm{e}^{i t \Delta} \rho \mathrm{e}^{-i t \Delta},
$$

so, as $\mathrm{e}^{i t \Delta}$ is unitary, $A(t) \in \mathcal{L}_{+}^{1}$ and $\|A(t)\|_{\mathcal{L}^{1}}=\|\rho\|_{\mathcal{L}^{1}}$ for every $t \in \mathbb{R}$. Note that $A(t)$ is $2 \pi \mathbb{Z}$-periodic in $t$.

When $\rho(x, y)=u(x) \overline{u(y)}$, the integral kernel of $\mathrm{e}^{i t \Delta} \rho \mathrm{e}^{-i t \Delta}$ is $\psi(x, t) \overline{\psi(y, t)}$ where $\psi(\cdot, t):=\mathrm{e}^{i t \Delta} u$, and $t_{\mathrm{e}^{i t \Delta} \rho \mathrm{e}^{-i t \Delta}}=\left|\mathrm{e}^{i t \Delta} u\right|^{2}$. Therefore, the dynamics of equation (2.11) reduce to those of (1.1) in that case.

For a given $\rho \in \mathcal{L}_{+}^{1}$, we shall denote by $b_{\rho}$ the Fourier coefficients of $t_{\mathrm{e}^{i t \Delta} \rho \mathrm{e}^{-i t \Delta}}$ :

$$
t_{\mathrm{e}^{i t \Delta} \rho \mathrm{e}^{-i t \Delta}}(x)=\sum_{(l, s) \in \mathbb{Z}^{d} \times \mathbb{Z}} b_{\rho}(l, s) \mathrm{e}^{i(l, s) \cdot(x, t)} .
$$

We now introduce some special classes of initial data in $\mathcal{L}_{+}^{1}$. Let $\Lambda$ be a submodule of $\mathbb{Z}^{d}$, let rk $\Lambda$ denote the rank of $\Lambda$. We define $\mathcal{L}_{+}^{1}(\Lambda)$ as the set consisting of the $\rho \in \mathcal{L}_{+}^{1}$ such that the integral kernel of $\rho$ is invariant by translations in directions in $\Lambda^{\perp}$ (this is the linear subspace orthogonal to $\Lambda$ ); in other words:

$$
\rho(x+v, y+w)=\rho(x, y), \quad \forall v, w \in \Lambda^{\perp} .
$$

Clearly, the classes $\mathcal{L}_{+}^{1}(\Lambda)$ are invariant by the dynamics of (2.11). The following result holds.

Theorem 2.2. Let $\Lambda \subset \mathbb{Z}^{d}$ be a submodule with $\mathrm{rk} \Lambda>0$. Then there exists a constant $C_{\mathrm{rk} \Lambda}>0$ such that:

$$
\left\|b_{\rho}\right\|_{l^{\mathrm{rk} \Lambda+1}\left(\mathbb{Z}^{d+1}\right)} \leq C_{\mathrm{rk} \Lambda}\left\|t_{\rho}\right\|_{L^{1}\left(\mathbb{T}^{d}\right)}
$$

for every $\rho \in \mathcal{L}_{+}^{1}(\Lambda)$.

Proof. Let $\left(u_{n}\right)$ be an orthonormal basis of $L^{2}\left(\mathbb{T}^{d}\right)$ consisting of eigenfunctions of $\rho$. One has

$$
\rho(x, y)=\sum_{i=1}^{\infty} \lambda_{n} u_{n}(x) \overline{u_{n}(y)}
$$

with $\lambda_{n} \geq 0, n \in \mathbb{N}$, and $\sum_{n=1}^{\infty} \lambda_{n}=\operatorname{Tr}(\rho)=\left\|t_{\rho}\right\|_{L^{1}\left(\mathbb{T}^{d}\right)}$. Clearly,

$$
b_{\rho}=\sum_{n=1}^{\infty} \lambda_{n} b_{u_{n}}
$$

moreover, since $\rho$ satisfies (2.12) and $\Lambda$ is a submodule of $\mathbb{Z}^{d}$ necessarily each $u_{n}$ is of the form:

$$
u_{n}(x)=\sum_{k \in \Lambda} a_{k}^{n} \mathrm{e}^{i k \cdot x},
$$

and $b_{u_{n}}(l, s)=0$ whenever $l \notin \Lambda$. Note moreover that for $l \in \Lambda \backslash\{0\}$ one has that $\Lambda \cap H_{(l, s)}$ spans an affine variety of dimension strictly less than $\operatorname{rk} \Lambda$ (since $\{n l\}_{n \in \mathbb{Z}}$ 
is not contained in $\left.H_{(l, s)}\right)$. This implies that $\left(\Lambda \cap H_{(l, s)}\right)^{\mathrm{rk} \Lambda+1} \cap \mathcal{V}_{\mathrm{rk} \Lambda}^{\mathrm{rk} \Lambda}=\emptyset$ and

$$
\left|b_{u_{n}}(l, s)\right|^{\mathrm{rk} \Lambda+1} \leq \sum_{r=0}^{\mathrm{rk} \Lambda-1} \sum_{\left(j_{1}, \ldots, j_{\mathrm{rk} \Lambda+1}\right) \in\left(\Lambda \cap H_{(l, s)}\right)^{\mathrm{rk} \Lambda+1} \cap \mathcal{V}_{r}^{\mathrm{rk} \Lambda}} \prod_{\sigma=1}^{\mathrm{rk} \Lambda+1}\left|a_{j_{\sigma}+l}^{n} a_{j_{\sigma}}^{n}\right| .
$$

With this in mind, one can apply Lemma 2.1 with $\delta=\operatorname{rk} \Lambda$ to conclude, as in Theorem 1.1:

$$
\sum_{\substack{(l, s) \in \mathbb{Z}^{d+1} \\ l \in \Lambda}}\left|b_{u_{n}}(l, s)\right|^{\mathrm{rk} \Lambda+1}=1+\sum_{r=0}^{\mathrm{rk} \Lambda-1} T_{r, \operatorname{rk} \Lambda}\left(u_{n}\right) \leq 2^{\mathrm{rk} \Lambda} .
$$

Therefore, using (2.13) we obtain the estimate:

$$
\left\|b_{\rho}\right\|_{l^{\mathrm{rk} \Lambda+1}\left(\mathbb{Z}^{d+1}\right)} \leq \sum_{n=0}^{\infty} \lambda_{n}\left\|b_{u_{n}}\right\|_{l^{\mathrm{rk} \Lambda+1}\left(\mathbb{Z}^{d+1}\right)} \leq C_{\mathrm{rk} \Lambda} \sum_{n=0}^{\infty} \lambda_{n}=C_{\mathrm{rk} \Lambda}\left\|t_{\rho}\right\|_{L^{1}\left(\mathbb{T}^{d}\right)}
$$

\section{Regularity of the limits}

We start by giving the proof of Theorem 1.2, which relies on Theorem 2.2 and the results from $[2]$.

3.1. Proof of Theorem 1.2. Without loss of generality, we can assume that (1.6) holds for the whole sequence $\left(u_{n}\right)$. Let $\left(h_{n}\right)$ the sequence appearing in property $(\mathbf{S})$. Denote by $\nu$ the weak-* limit of $\left(\left|\mathrm{e}^{i t \Delta} u_{n}\right|^{2}\right)$. Theorem 3 in [2] ensures that,

$$
\nu(x, t)=\sum_{\Lambda} t_{\mathrm{e}^{i t \Delta} \rho_{\Lambda} \mathrm{e}^{-i t \Delta}}(x)
$$

where the sum ranges over all (primitive) submodules of $\mathbb{Z}^{d}$, and the operators $\rho_{\Lambda} \in$ $\mathcal{L}_{+}^{1}(\Lambda)$ only depend on the sequence of initial data $\left(u_{n}\right)$. Because the functions $t_{\rho_{\Lambda}}$ are non-negative, we have:

$$
\sum_{\Lambda}\left\|t_{\rho_{\Lambda}}\right\|_{L^{1}\left(\mathbb{T}^{d}\right)}=\sum_{\Lambda}\left\|t_{\mathrm{e}^{i t \Delta} \rho_{\Lambda} \mathrm{e}^{-i t \Delta}}\right\|_{L^{1}\left(\mathbb{T}^{d}\right)}=\nu\left(\mathbb{T}^{d}, t\right) \leq \limsup _{n \rightarrow \infty}\left\|u_{n}\right\|_{L^{2}\left(\mathbb{T}^{d}\right)}^{2} .
$$

We claim that if hypothesis $(\mathbf{S})$ holds, then $\rho_{\mathbb{Z}^{d}}=0$. In that case, only submodules of rank strictly less than $d$ appear in the sum (3.1). Therefore, we can apply Theorem 2.2 and find:

$$
\|\widehat{\nu}\|_{l^{d}\left(\mathbb{Z}^{d+1}\right)} \leq \sum_{\Lambda}\left\|b_{\rho_{\Lambda}}\right\|_{l^{d}\left(\mathbb{Z}^{d+1}\right)} \leq C \sum_{\Lambda}\left\|t_{\rho_{\Lambda}}\right\|_{L^{1}\left(\mathbb{T}^{d}\right)}<\infty .
$$

We finally show that $\rho_{\mathbb{Z}^{d}}=0$. Hypothesis $(\mathbf{S})$ implies that any limit $\mu_{0} \in \mathcal{M}_{+}\left(\mathbb{T}^{d} \times \mathbb{R}^{d}\right)$ of the sequence of Wigner distributions $w_{u_{n}}^{h_{n}}$ (in the notation of [2]) satisfies:

$$
\mu_{0}\left(\mathbb{T}^{d} \times\{0\}\right)=0 .
$$

The reader may find the standard argument in, for instance, [7]. One can then apply Corollary 30 in [2] and conclude the proof. 
3.2. Additional regularity results. It is possible to replace $(\mathbf{S})$ by a family of stronger conditions that ensure additional regularity on the weak-* accumulation points of orbits of the Schrödinger flow. In order to introduce them we must recall some notations from [2].

Denote by $\Omega_{j} \subset \mathbb{R}^{d}$, for $j=0, \ldots, d$, the set of resonant vectors of order exactly $j$, that is:

$$
\Omega_{j}:=\left\{\xi \in \mathbb{R}^{d}: \operatorname{rk} \Lambda_{\xi}=d-j\right\}
$$

where $\Lambda_{\xi}:=\left\{k \in \mathbb{Z}^{d}: k \cdot \xi=0\right\}$. A classical result shows that $\xi \in \Omega_{j}$ if and only if for any $x \in \mathbb{T}^{d}$ the geodesic $\tau \mapsto x+\tau \xi$ is dense in a subtorus of $\mathbb{T}^{d}$ of dimension $j$.

For each $r=1, \ldots, d+1$ we introduce the following condition $\left(\mathbf{S}_{r}\right)$ on the sequence of initial data $\left(u_{n}\right)$ :

$\left(\mathbf{S}_{r}\right)$ There exists a sequence of positive reals $\left(h_{n}\right)$ tending to zero such that $(1.8)$ holds and every accumulation point $\mu_{0} \in \mathcal{M}_{+}\left(\mathbb{T}^{d} \times \mathbb{R}^{d}\right)$ of the sequence of Wigner distributions $\left(w_{u_{n}}^{h_{n}}\right)$ (as defined in [2]) satisfies:

$$
\mu_{0}\left(\mathbb{T}^{d} \times \bigcup_{j<r} \Omega_{j}\right)=0
$$

Note that the condition $(\mathbf{S})$ is equivalent to $\left(\mathbf{S}_{1}\right)$; moreover, $\left(\mathbf{S}_{d+1}\right)$ is equivalent to the fact that $\left(u_{n}\right)$ converges strongly to 0 in $L^{2}\left(\mathbb{T}^{d}\right)$. Condition $\left(\mathbf{S}_{r}\right)$ roughly states that no fraction of the $L^{2}$-norm of the $u_{n}$ concentrates on resonances of order strictly less than $r$ as $n \rightarrow \infty$.

In [9] it is shown that whenever $\left(\mathbf{S}_{d}\right)$ holds, we have that every weak-* accumulation point is constant.

The following generalization of Theorem 1.2 is true.

Theorem 3.1. Suppose that $\left(u_{n}\right)$ is a bounded sequence in $L^{2}\left(\mathbb{T}^{d}\right)$ that satisfies condition $\left(\boldsymbol{S}_{r}\right)$ for some $r=1, \ldots, d-1$. Let $\nu \in L^{\infty}\left(\mathbb{T} ; L^{1}\left(\mathbb{T}^{d}\right)\right)$ be a weak-* accumulation point of $\left(\left|\mathrm{e}^{i t \Delta} u_{n}\right|^{2}\right)$. Then:

$$
\|\widehat{\nu}\|_{l^{d+1-r}\left(\mathbb{Z}^{d+1}\right)}<\infty
$$

The proof of this result follows exactly the lines of that of Theorem 1.2; except that hypothesis $\left(\mathbf{S}_{r}\right)$ is now used, via Corollary 30 in [2], to ensure that $\rho_{\Lambda}=0$ for every primitive submodule $\Lambda \subset \mathbb{Z}^{d}$ of rank strictly greater than $d-r$.

\section{Acknowledgments}

The authors would like to thank Patrick Gérard for pointing out an inaccuracy in a previous version of this paper. T.A. was supported by FQRNT. D.J. was supported by NSERC, FQRNT and Dawson fellowship. F.M. was supported by grant MTM201016467 (MEC), and wishes to acknowledge the support of ICMAT through its visiting faculty program.

\section{References}

[1] T. Aïssiou, Semiclassical limits of eigenfunctions on flat $n$-dimensional tori, Can. Math. Bull. (2011) To appear.

[2] N. Anantharaman and F. Macià, Semiclassical measures for the Schrödinger equation on the torus (2010), arXiv:1005.0296v2. 
[3] J. Bourgain, Fourier transform restriction phenomena for certain lattice subsets and applications to nonlinear evolution equations. I. Schrödinger equations, Geom. Funct. Anal. 3(2) (1993), 107-156.

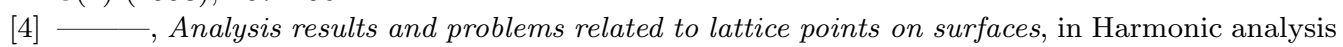
and nonlinear differential equations (Riverside, CA, 1995), Contemp. Math. 208, 85-109, Amer. Math. Soc., Providence, RI (1997).

[5] - Moment inequalities for trigonometric polynomials with spectrum in curved hypersurfaces (2011), arXiv:1107.1129v1.

[6] R. Cooke, A Cantor-Lebesgue theorem in two dimensions, Proc. Amer. Math. Soc. 30 (1971), $547-550$.

[7] P. Gérard, Description du défaut de compacité de l'injection de Sobolev, ESAIM Control Optim. Calc. Var. 3 (1998), 213-233 (electronic).

[8] D. Jakobson, Quantum limits on flat tori, Ann. Math. (2) 145(2) (1997), 235-266.

[9] F. Macià, Semiclassical measures and the Schrödinger flow on Riemannian manifolds, Nonlinearity 22(5) (2009), 1003-1020.

[10] F. Macià, High-frequency propagation for the Schrödinger equation on the torus, J. Funct. Anal. 258(3) (2010), 933-955.

[11] N. Nadirashvili, J. Toth, and D. Jakobson, Geometric properties of eigenfunctions, Russian Math. Surveys 56(6) (2001), 1085-1105.

[12] A. Zygmund, On Fourier coefficients and transforms of functions of two variables, Studia Math. 50 (1974), 189-201.

Department of Mathematics and Statistics, Concordia University, 1455 de Maisonneuve Blvd. West, Montreal, Quebec, H3G 1M8, Canada

E-mail address: aissiou@math.mcgill.ca

Department of Mathematics and Statistics, McGill University, 805 Sherbrooke Str. West, MontréAl QC H3A 2K6, CANADA

E-mail address: jakobson@math.mcgill.ca

DCAin, ETSi Navales, Universidad Politécnica de Madrid, Avda. Arco de la Victoria 4 28040 MADRID, SPAIN

E-mail address: fabricio.macia@upm.es 
